\title{
Ultra-rapid UT1 measurement by e-VLBI
}

\author{
Mamoru Sekido ${ }^{1}$, Hiroshi Takiguchi ${ }^{1}$, Yasuhiro Koyama ${ }^{1}$, Tetsuro Kondo ${ }^{1}$, Rüdiger Haas ${ }^{2}$, \\ Jan Wagner $^{3}$, Jouko Ritakari ${ }^{3}$, Shinobu Kurihara ${ }^{4}$, and Kensuke Kokado ${ }^{4}$ \\ ${ }^{1}$ National Institute of Information and Communications technology, Kashima Space Research Center, \\ 893-1, Hirai, Kashima, Ibaraki 314-8501, Japan \\ ${ }^{2}$ Chalmers University of Technology, Onsala Space Observatory, SE-439 92 Onsala, Sweden \\ ${ }^{3}$ Helsinki University of Technology, Metsähovi Radio Observatory, Metsähovintie 114 FIN-02540 Kylmälä, Finland \\ ${ }^{4}$ Geographical Survey Institute, 1 Kitasato, Tsukuba 305-0811, Japan
}

(Received October 31, 2007; Revised May 13, 2008; Accepted June 6, 2008; Online published September 8, 2008)

\begin{abstract}
The latency of UT1 measurement with Very Long Baseline Interferometry (VLBI) has been greatly reduced by using e-VLBI technology. VLBI observations on the baseline formed by the Kashima 34-m and the Onsala 20-m radio telescopes achieved ultra-rapid UT1 measurements, where the UT1 result was obtained within 30 min after the end of the observing session. A high speed network and a UDP-based data transfer protocol 'Tsunami' assisted the high data rate and long-distance data transfer from Onsala to Kashima. The accuracy of the UT1 value obtained from the $1-\mathrm{h}$ single baseline e-VLBI experiment has been confirmed to be as the same level with the rapid combined solution of Bulletin-A. The newly developed technology is going to be transferred to the regular intensive VLBI sessions, and it is expected to contribute to the improved latency and accuracy of UT1 data.
\end{abstract}

Key words: E-VLBI, UT1.

\section{Introduction}

The Earth Orientation Parameters (EOP) represent the irregularity of Earth rotation, and they provide the coordinate transformation between the International Terrestrial and Celestial Reference Frames (respectively, ITRF and ICRF) in conjunction with the conventional Precession-Nutation model (McCarthy and Petit, 2004). The EOP consists of polar motion and UT1. Progress is being made in our understanding of the excitation mechanism of polar motion and variation of UT1, although a prediction of them with sufficient accuracy is still difficult at present. Thus, EOP data have to be determined observationally by using space geodetic techniques, such as very long baseline interferometry (VLBI), the Global Satellite Navigation System (GNSS), and Satellite Laser Ranging (SLR).

The UT1 is the rotation angle of the Earth relative to the conventional origin of the ICRF, whose axis directions are fixed relative to the distant matter of universe (McCarthy and Petit, 2004). Therefore, the UT1 is essentially accessible only by astronomical observation of extragalactic objects. VLBI measures the arrival time differences of extragalactic radio signal between pairs of radio telescopes very precisely. The geometrical VLBI delay model is theoretically based on terrestrial time (TT), and Coordinated Universal Time (UTC) is practically used as time argument for computing the observation epoch in TT. Then VLBI can measure the irregularity of the earth's rotation angle with respect to the UTC, which corresponds to the value UT1-

Copyright (c) The Society of Geomagnetism and Earth, Planetary and Space Sciences (SGEPSS); The Seismological Society of Japan; The Volcanological Society of Japan; The Geodetic Society of Japan; The Japanese Society for Planetary Sciences; TERRAPUB
UTC. Currently, VLBI is the only one space geodetic technique that can monitor the UT1-UTC with long time stability.

Historically, global VLBI observations for Earth's rotation measurements have been performed within the framework of several projects: IRIS-A, IRIS-P, IRIS-S, NAVNET/NAVEX, and CDP (e.g. Ma and Caprette, 1992). Also, a series of single baseline special VLBI sessions named 'Intensive sessions' has been conducted on the Westford (Massachusetts, USA)-Wettzell (Bavaria, Germany) baseline since 1985 (Robertson et al., 1985; Carter et al., 1992). Currently, the single baseline 'Intensive sessions' are continuously operated with the aim of UT1 observation with minimum latency (Behrend and Nothnagel, 2007). An e-VLBI experiment conducted with the Kashima-Westford baseline (Koyama et al., 2004) achieved a UT1 measurement within $4.5 \mathrm{~h}$, and it demonstrated usefulness of the e-VLBI for quick UT1 measurement. After the success of the experiment, FTP-based data transfer began to be used in the Intensive sessions. It has improved the latency by about 3 days relative to the earlier approach (Machida et al., 2006), where recorded magnetic media used to be transported as parcels. That improvement of the latency has consequently contributed the prediction accuracy of EOP in Rapid-Service (see Section 4) by 20-47\% (Johnson et al., 2005).

The aim of our project is to utilize e-VLBI technology to perform UT1 measurements as quick as possible. The technology developed in this project will be transferred to regular intensive sessions and will contribute to an improvement in the latency and accuracy of UT1 data. This paper reports the achievement of ultra rapid UT1 measurements 
with real-time data transport and quick data processing. In the following section, technical details of the e-VLBI experiments are described. Observation parameters and data analysis are described in Section 3. Evaluation of the UT1 data accuracy is discussed in Section 4.

\section{Technical Details of E-VLBI Experiments}

E-VLBI, which is optically linked VLBI for real-time or near-real-time VLBI (Whitney, 2000), has become popular by taking advantage of recent progress in network technology and network infrastructure. Numerous projects connecting radio telescopes with high speed network are in progress (e.g. Alef, 2007; Tingay et al., 2007). One of the key factors to enable the rapid output of results in a VLBI observation is the choice of data transport protocol. The data rate of VLBI observation is as large as 256 Mega bit per seconds (Mbps) or more, and the total amount of data reaches more than 1.4 Tera-Byte at one station. Thus, highspeed data transport from the observation site to the correlation center is required at a rate as fast as the data acquisition. Additionally, the preservation of phase information of the received radio signal is essential for interferometry. For this reason, time tag information assigned to each samples of the data has to be preserved in the data transportation. $\mathrm{TCP} / \mathrm{IP}$ is a protocol to guarantee the reliable data transport over the network. It is widely used for application, which requires rigorous data transfer. However a drawback of TCP/IP is data transport slowdown over a long-distance network. It is known that the data transfer rate of TCP/IP is theoretically limited by the relation

$$
\text { rate }[\mathrm{bps}]=8 \times(\text { Windows size }[\text { Byte }]) /(\text { RTT }[\mathrm{sec}]),
$$

where, RTT is the round trip time between end-to-end of the network (e.g. Hirabaru, 2004). Thus, the transmission rate slows down at a rate inversely proportional to the RTT over long distance. This limitation comes from the acknowledgment mechanism inherent to TCP/IP, which ensures the rigorous packet delivery in communications.

The UDP/IP based protocol, 'Tsunami', was developed by the Advanced Network Management Laboratory of Indiana University as an experimental high speed network file transfer protocol over very long distances (Meiss, 2004). Wagner and Ritakari of the Metsähovi Radio Observatory applied it for real-time data transfer from their VLBI interface card. This protocol can achieve a data rate of more than $600 \mathrm{Mbps}$ in data transfer at several thousands $\mathrm{km}$ distance of 1-Gbps network, where standard TCP/IP could achieve a data rate of only around $32 \mathrm{Mbps}$. This "Tsunami" protocol was used for our e-VLBI experiments. The location of VLBI stations (Kashima, Onsala, Tsukuba and Metsähovi) participating in this Ultra-rapid UT1 experiments are indicated on the map (Fig. 1). Accessible network speed was $1 \mathrm{Gbps}$ for Kashima, Tsukuba, Onsala, and $10 \mathrm{Gbps}$ for Metsähovi. The Mark5 VLBI data acquisition system (DAS) developed by the Haystack Observatory (Whitney, 2004; Haystack Observatory, 2006) was used in the Onsala and Metsähovi stations. The data captured by Mark5 data were fed to VSI board (VSIB), which was developed by the Metsähovi observatory (Ritakari and Mujunen, 2004). The data were then sent out to the network in real-time.

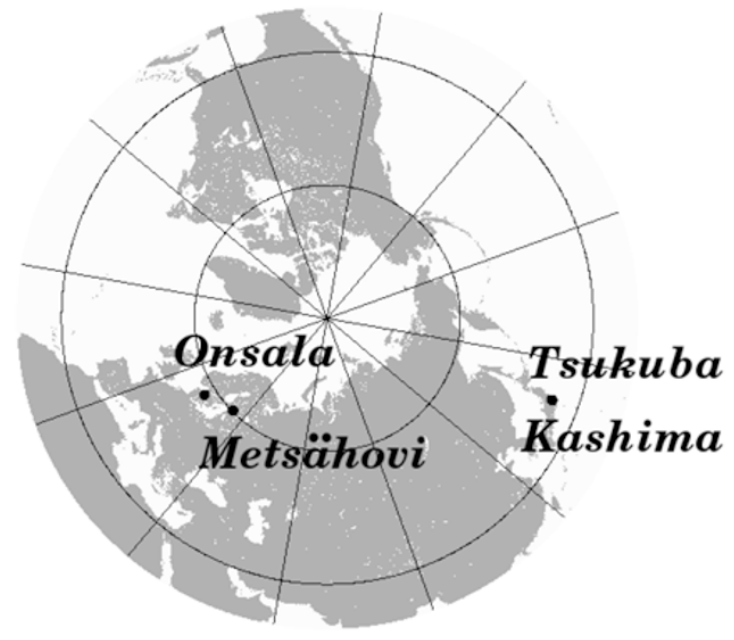

Fig. 1. VLBI stations participating in the ultra-rapid UT1 experiments.

The K5/VSSP32 VLBI-DAS developed by NICT (Kondo et al., 2006) was used for observation at the Kashima and Tsukuba stations. Total data rate on the VLBI observation was $128 \mathrm{Mbps}$ or $256 \mathrm{Mbps}$ (see Table 1). The data transfer from Onsala or Metsähovi station to Japan was made in real-time during the observation. After the end of each scan, the Mark5 data observed at Nordic stations were converted to the K5/VSSP32 data format automatically. Then correlation processing jobs were shared by a cluster of computers in parallel. The data format conversions and the correlation processing were performed by a software package developed by $\mathrm{NICT}^{* 1}$ (Kondo et al., 2004). An improvement in the compatibility among different types of DASs is another benefit of e-VLBI. By using this e-VLBI system, correlation processing is finished $5 \mathrm{~min}$ after the session, and the UT1-UTC is derived within $30 \mathrm{~min}^{* 2}$ thereafter. It is notable that the shared network was used in this e-VLBI experiments without any special arrangements. The network traffic from the Nordic stations to Kashima has come through several international and domestic networks, such as SUNET, Funet, NORDUnet, DANTE/GÈANT2, Internet2/Abilene, JGN2, and SINET3. These high-speed networks are freely available for scientific and for technology development purposes. Thanks to these inter-connected high speed networks, we need not be aware of the network route between the source and destination.

\section{UT1 Observations with VLBI}

The diameters of the telescopes which participated in the experiments and their sensitivity parameters are listed in Table 2. Our e-VLBI experiments on the UT1 measurement have been operating since April 2007. The observation schedule is important in geodetic VLBI, since the precision of UT1-UTC measurement and its covariance matrix depends on the observation schedule, which describes the sequence of observing radio sources and duration for

\footnotetext{
${ }^{* 1}$ http://www2.nict.go.jp/w/w114/stsi/K5/VSSP/index-e.html. This package is freely available by license agreement.

${ }^{* 2}$ After the submission of this paper, further quick processing was successfully achieved in February 2008, where UT1 data was obtained within 4
} min after the session. 
Table 1. E-VLBI sessions for rapid UT1 measurement performed since April 2007. Station codes are as follows, 'Ks': Kashima34, 'Ts': Tsukuba 32, 'On': Onsala, 'Mh': Metsähovi, 'Wz': Wettzell. The effective band widths for the experiments from 3 April to 4 June were 140.2 MHz and 33.1 MHz for the X and S-band, respectively, for experiments from 14 and 15 July were respectively $280.4 \mathrm{MHz}$ and $48.8 \mathrm{MHz}$ for the X and S-band. The experiments on 14 and 15 July were performed by add on to the intensive session (INT-2) of Tsukuba-Wettzell baseline.

\begin{tabular}{|c|c|c|c|c|c|c|}
\hline $\begin{array}{l}\text { Date } \\
2007\end{array}$ & Baseline & $\begin{array}{c}\text { Data rate } \\
(\mathrm{Mbps})\end{array}$ & $\begin{array}{l}\text { UT1-UTC } \\
(\mathrm{ms})\end{array}$ & $\begin{array}{c}\text { UT1-c04 } \\
(\mu \mathrm{s})\end{array}$ & $\begin{array}{c}\text { Error } \\
(\mu \mathrm{s})\end{array}$ & Latency \\
\hline 03 April & Ks-On & 256 & -69.6044 & -38.5 & 8 & - \\
\hline 23 April & Ks-On & 128 & -98.4422 & 15.0 & 41 & $1 \mathrm{~h} 55 \mathrm{~min}$. \\
\hline 02 May & Ks-On & 128 & -110.0189 & -30.4 & 16 & 一 \\
\hline 18 May & Ks-Mh & 128 & -130.5832 & 67.5 & 98 & 2 h $38 \mathrm{~min}$. \\
\hline 30 May & $\mathrm{Ks}-\mathrm{On}$ & 128 & -143.2703 & -14.7 & 9 & $28 \mathrm{~min}$. \\
\hline 31 May & Ks-On & 128 & -143.7011 & -83.5 & 8 & - \\
\hline 04 June & Ks-On & 256 & -144.6447 & 13.1 & 6 & $31 \mathrm{~min}$. \\
\hline \multirow[t]{7}{*}{14 July } & Ks-On, Ks-Wz & 256 & -162.0879 & 6.2 & 6 & - \\
\hline & Ks-On & & -162.1017 & -7.6 & 10 & \\
\hline & $\mathrm{Ks}-\mathrm{Wz}$ & & -162.0715 & 22.6 & 8 & \\
\hline & Ts-Wz, On-Ts & & -162.0674 & 26.7 & 8 & \\
\hline & On-Ts & & -162.0725 & 21.6 & 7 & \\
\hline & Ts-Wz & & -162.0585 & 35.6 & 5 & \\
\hline & Ts-Wz (INT2) & & -162.0974 & -3.3 & 7 & \\
\hline \multirow[t]{2}{*}{15 July } & Ks-On & 256 & -162.0186 & -30.7 & 6 & - \\
\hline & Ts-Wz (INT2) & & -162.0017 & -13.8 & 8 & \\
\hline
\end{tabular}

each sources. Observation schedules were created by using automatic schedule generation function of geodetic VLBI scheduling software 'sked' (Gipson, 1999), where the optimizing option for UT1 and minimum SNR setting to 20 and 15 were used for the X-band and S-band, respectively. The duration of the observation session was $1 \mathrm{~h}$ which is as long as IVS intensive sessions. Before the main session, we added $30 \mathrm{~min}$ of observation for fringe finding, which is a procedure to fix the clock parameters by using the observation of strong radio sources. The clock parameters represent the synchronization difference between atomic clocks at VLBI stations, and these parameters have to be fixed prior to the correlation processing. When observations are routinely performed, the clock difference can be estimated with sufficient accuracy from the prior observation. In this case, this procedure may be skipped. After the correlation processing, precise group delay observables were derived with bandwidth synthesis procedure (Rogers, 1970). The data were then stored in the form of Mark3 database, and the UT1-UTC parameter was derived with the CALC/SOLVE package, which was developed by VLBI geodetic group of NASA/GSFC (Petrov, 2007). In addition to the UT1UTC, atmospheric parameters (0th-order) for both stations and clock parameters (0th and 1st-order) were estimated in the analysis. Table 1 shows the UT1-UTC results obtained by the series of e-VLBI sessions performed since April in 2007. Since the automatic processing environment was not ready in the early stage, it took a few days to derive the analysis results. Finally, since the end of May 2007, it has become possible to measure the UT1-UTC within $30 \mathrm{~min}$. The latency of UT1 measurement has been improved remarkably over the former record, which was $4.5 \mathrm{~h}$ of latency achieved in 2004 by Koyama et al. (2004) on the KashimaWestford baseline. The indication '- ' in the 'Latency' in the Table 1 means that the data were processed offline instead of being processed in near-real-time. This was mainly because of the time-consuming fringe search process to fix
Table 2. Diameter and system equivalent flux density (SEFD) of radio telescopes.

\begin{tabular}{cccc}
\hline \multirow{2}{*}{ Station name } & Diameter & \multicolumn{2}{c}{ SEFD (Jy) } \\
\cline { 3 - 4 } & $(\mathrm{m})$ & X-band & S-band \\
\hline Kashima 34 & 34 & 380 & 430 \\
Tsukuba 32 & 32 & 320 & 360 \\
Onsala 60 & 20 & 1630 & 1110 \\
Metsähovi & 13.7 & 3200 & 4500 \\
\hline
\end{tabular}

clock parameters mentioned above. However this will not be an essential problem in regular sessions where clock parameters are easily estimated from the latest session less than 1 week before. Currently, details of the 30-min delay are mostly spent for creating the Mark3 database and interactive analysis with CALC/SOLVE. Correlation processing is completed within 5 min after the session. Further improvements of latency may be possible by automation of the database creation and automatic analysis*3. Such a procedure is being developed by T. Hobiger as database software 'Mk3tools' (Hobiger et al., 2007) and as an automatic iterative analysis procedure with VLBI data analysis software OCCAM (Titov et al., 2004). The details and achievement of the further improvement of latency in UT1 observation will be published in the near future.

\section{Discussion}

Several agencies as analysis centers of space geodetic techniques (VLBI, SLR, and the GPS) regularly produce EOP series and submit them to IERS. The combined solution by IERS is published in IERS Bulletin-B every month, and that data cover the epoch up to 1 month before the date of issue. For users who need EOP data at current or future epochs, prediction values of EOP covering 1 year in the fu-

\footnotetext{
${ }^{* 3}$ Quick UT1 measurement within 4 min in February 2008 was achieved with these softwares.
} 
(a)

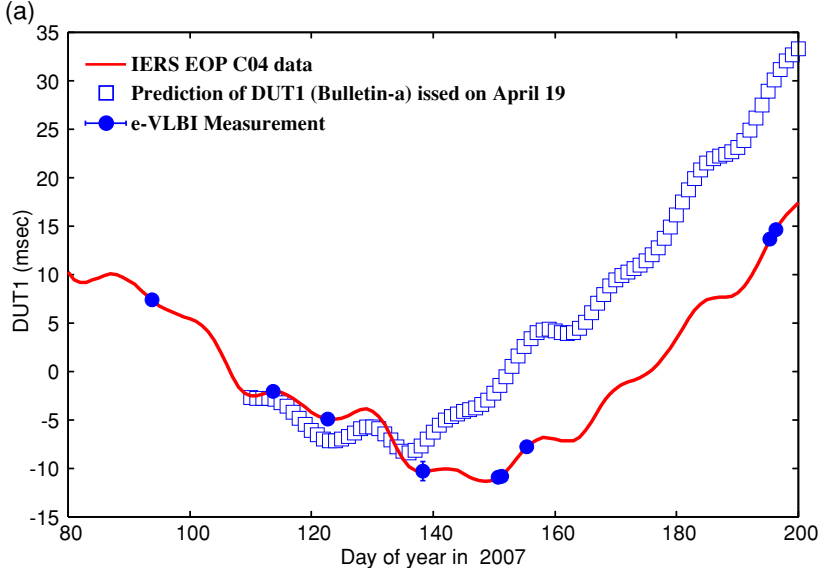

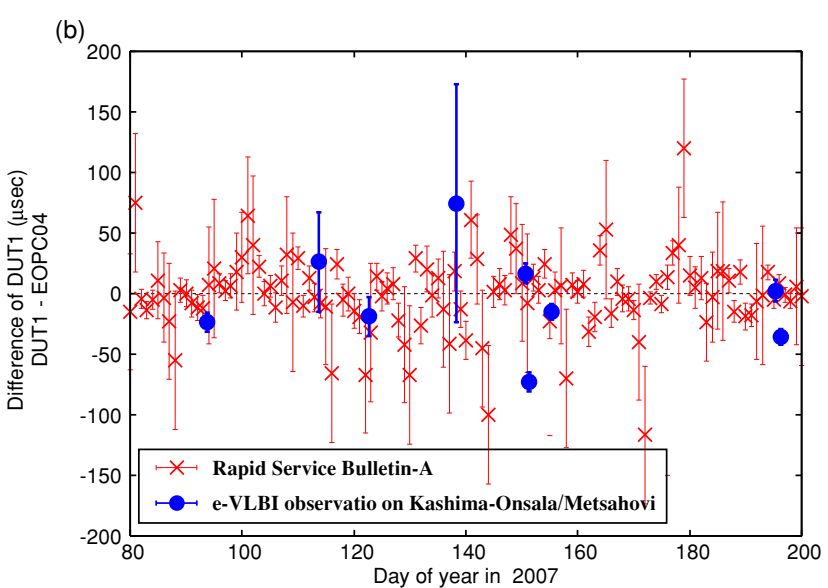

Fig. 2. UT1-UTC values measured by e-VLBI ('•’), IERS EOPc04 (solid line) and UT1 prediction value of Bulletin-A (' $\square$ ') are plotted in the left plot, where the linear trend is subtracted. Prediction values are taken from Bulletin-A on the 19 April issue (Day of year = 109). Right plot: UT1-UTC data of e-VLBI measurement (' $\bullet$ ') and those of Rapid-Service of Bulletin-A (' $\times$ ') are plotted after subtraction of the EOPc04 series. The error bars are the formal error for e-VLBI and uncertainty for the Rapid-Service.

ture are published weekly as IERS Bulletin-A (IERS, 2004). For a compensation of the poor accuracy of prediction data in Bulletin-A and latency of Bulletin-B, rapid combined solutions (Rapid-Service) are also published weekly. These are derived by combining the latest EOP solution with data of VLBI, SLR, GPS, and meteorological predictions of Atmospheric Angular Momentum (AAM). Thus, a rapid turn around of UT1 measured by VLBI can contribute to the improvement of UT1 accuracy. The UT1-UTC measured by e-VLBI, prediction data of Bulletin-A, and IERS-EOPc04 series are plotted in Fig. 2. Hereafter we use the EOPc04, which is one of the best estimates of true EOP series, as reference for comparisons. The left plot of Fig. 2(a) clearly shows that the prediction accuracy degrades as time increases from the date of issue, whereas the UT1-UTC data measured by e-VLBI coincide with the EOPc04. The right plot of Fig. 2(b) shows the comparison between e-VLBI data and the Rapid-Service, which has an accuracy of about a few tens of microseconds. The plot indicates that UT1UTC measurements by e-VLBI have the same accuracy with the Rapid-Service. Although the Rapid-Service covers the period from 1 week before to the date of issue, it cannot be used for real-time application. For a clear understanding of the accuracy of UT1-UTC data available for real-time application, the best estimates of UT1-UTC data available at each epoch are displayed in Fig. 3 for the period from the 80th to 200th day of the year (DOY) in 2007. The figure shows the deviation of UT1-UTC data from EOPc04 series for prediction, Rapid-Service, and measurements by e-VLBI. The prediction data were picked up from BulletinA data, which were available at the moment of each epoch. The figure indicates that the deviation of the prediction data increases with time, then it becomes small at every 7th day when new Bulletin-A data are published. Root-meansquare (RMS) of the difference of UT1-UTC between prediction and EOPc04 data is $0.4 \mathrm{~ms}$ for this period. This is not sufficient for some applications. For example, spacecraft navigation requires 1 nano radian $(=13 \mu$ s of time) to use air-drag braking at the Mars, which enables a drastic reduction of fuel requirement in spacecraft.

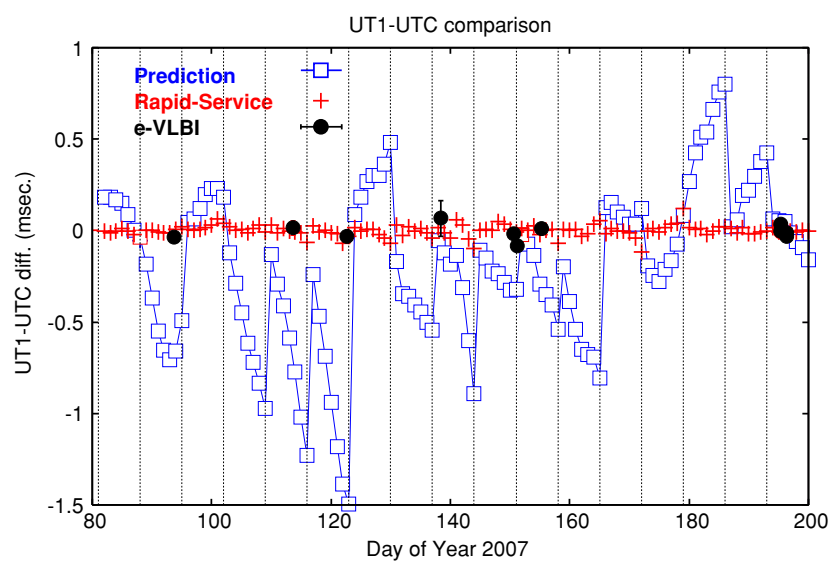

Fig. 3. Available UT1-UTC prediction data of Bulletin-A for real-time applications are plotted with the Rapid-Service and e-VLBI measurements data, where data of IERS EOPc04 are subtracted from each series as a reference. Since Bulletin-A is published every week, the deviation increase with time then it reduces at every 7 th day. Vertical dotted lines indicate the epochs, when data of Bulletin-A are published. The RMS of deviation of prediction, the Rapid-Service, and e-VLBI from EOPc04 were respectively $0.4 \mathrm{~ms}, 30 \mu \mathrm{s}$, and $34 \mu \mathrm{s}$ for the period from 80 to 200 day of year in 2007.

It is not easy to estimate quantitatively how much the accuracy of the EOP prediction value could be improved by the contribution of our e-VLBI technique-prior to its implementation into regular intensive sessions. One of the estimates of accuracy improvement of the UT1-UTC prediction will be obtained by shifting the error characteristic of the UT1-UTC prediction. Figure 4 indicates uncertainty of UT1-UTC published by Bulletin-A and B. This plot is a hybrid of the log-log plot for future (right half from the center) and semi-log plot for the past (left half from the center). The envelope lines represent the uncertainties of UT1-UTC in each region, which are taken from the explanatory document of Bulletin-A/B (IERS, 2004). Plots of ' $x$ ' marks with solid lines in the 'future' and 'past' regions are the RMS deviation from EOPc04 computed for the prediction, and Rapid-Service data, respectively. The agreement be- 


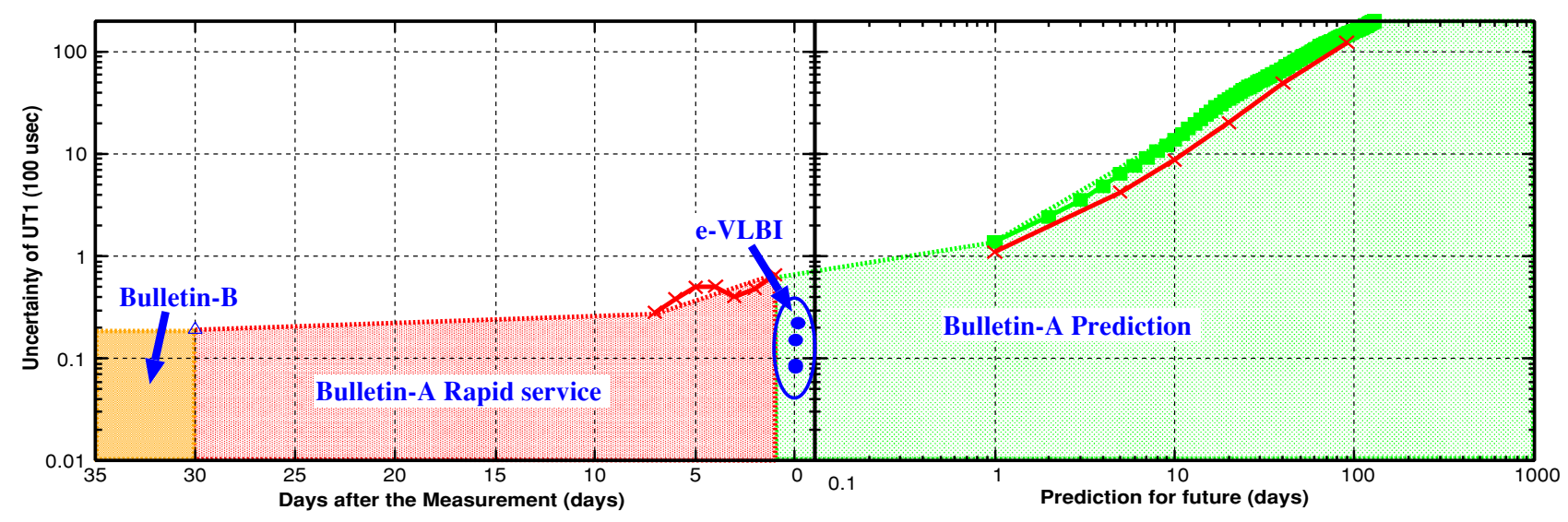

Fig. 4. Overview of UT1 data uncertainty is indicated with a hybrid-plot. Center is current time, left is past, and right is future. Left half is the semi-log plot of uncertainty versus time for the past. Right half is log-log plot for the future. Envelope is the uncertainty of UT1 for prediction, Rapid-Service, and Bulletin-B at each time region. The value of uncertainty is taken from a explanatory document of Bulletin-A/B. Plots of ' $x$ ' connected with solid lines in the 'past' and 'future' regions are root-mean-square (RMS) of deviation from EOPc04 series for prediction and Rapid-Service of UT1 data. The RMS are computed for the period from January 1 in 2000 to August 31 in 2007.

tween RMS and the envelope indicates that the error evaluation of the explanatory document is correct. Currently, VLBI intensive sessions are processed with a few days of delay between VLBI observation and output of EOP solution. If the UT1-UTC is measured in near-real-time by using our e-VLBI technique, 2-3 days of advancement will be gained. If we assume the prediction accuracy of UT1 is dominated only by the delay of the latest VLBI observation data, 3 days of advantage corresponds to an improvement of UT1-UTC uncertainty from $80 \mu \mathrm{s}$ to $40 \mu \mathrm{s}$ for 'present time'. This estimation may be too optimistic, since the process of computing UT1-UTC prediction data is not only dominated by a delay of intensive VLBI observation data, but also by length of day (LOD) data by GPS and AAM data. However, it is worth obtaining the order of accuracy improvement to be anticipated.

The other way of estimating accuracy improvement may be given by the experience of contribution by using current FTP-based e-VLBI. Johnson et al. (2005) reported that there was a small improvement in short-term UT1-UTC prediction, mainly due to e-VLBI intensive session. This is understandable from the slope of the 'past time' region of Fig. 4. Even at the moment of 1 month later, when almost sufficient observation data from space geodetic techniques and other data, such as LOD and AAM, become available, the improvement of uncertainty does not reduce to one order smaller. One of the causes of that may be remaining inconsistency among different space geodetic techniques in the Earth's orientation parameters. For instance, the comparison of EOP among VLBI analysis center shows agreement of UT1-UTC in the order of several $\mu$ s, whereas the uncertainty of UT1 is about $20 \mu \mathrm{s}$ in the combined solution. This inconsistency among space geodetic techniques should be reduced in the near future.

\section{Conclusion}

An ultra-rapid UT1 measurement has been made using the e-VLBI technology, and the UT1-UTC measurement has become available within $30 \mathrm{~min}$ after a VLBI observation. This has been achieved by using several advan- tages of e-VLBI technology: using UDP-based protocol, which enables high-speed data transfer over very long distances, automation of data processing, and enhancement of compatibility between different VLBI-DASs. The accuracy of our UT1-UTC estimation of our experiments was at the same level with Rapid-Service of Bulletin-A. The RMS difference and averaged offset between e-VLBI and EOPc04 series were, respectively, $34 \mu \mathrm{s}$ and $-0.9 \mu \mathrm{s}$.

It is notable that no special arrangements are required for these e-VLBI experiments over the continental baseline. The tools used for this experiments should be applicable at any other baseline where high-speed network access is available. The improvement of latency by this e-VLBI technique is about 2-3 days, and the corresponding prediction accuracy improvement for the real-time user was estimated as $50 \%$ at maximum.

Acknowledgments. We thank the network research community of SUNET, Funet, NORDUnet, DANTE/GÈANT, Internet2/Abilene, JGN2, and APAN for providing freely available high speed network connections between VLBI stations in Japan and Europe. We acknowledge T. Hobiger of NICT for the development of database creation software package "Mk3Tools". Takatoshi Ikeda, Hiroaki Harai, and Masaki Hirabaru in the Network Architecture Group of NICT have been supporting the eVLBI experiments. We thank the Finish Geodetic Institute for providing the S/X receiver for Metsähovi. We thank Gerhard Kronschnabl at Wettzell station in Germany for contributions in observations. Finally, we acknowledge the referees and editor of EPS for valuable comments which improved the manuscript.

\section{References}

Alef, W., E-VLBI Status at the MPIfR, 6th International e-VLBI workshop, http://www.mpifr-bonn.mpg.de/div/vlbi/6th_evlbi/, 2007.

Behrend, D. and A. Nothnagel, 3.4.3 International VLBI Service (IVS), IERS Annual Report 2006, 46-50, 2007.

Carter, W. E., D. S. Robertson, and J. R. Ray, Earth orientation time series derived from VLBI observations, IERS Tech. Note, 11, 33-39, 1992.

Gipson, J., GSFC VLBI SKED Software Package, http://lupus.gsfc.nasa. gov/software_sked.htm, 1999.

Haystack Observatory, Mark5/e-VLBI News Letter, http://www.haystack. edu/tech/vlbi/mark5/newsletter.html, 8, 2006.

Hirabaru, M., Performance Measurement on Large Bandwidth-Delay Product Network, Proceedings of the 3rd International e-VLBI work- 
shop, IVS NICT-TDC News, http://www2.nict.go.jp/w/w114/stsi/ivstdc/ news-index.html, 25, 11-19, 2004.

Hobiger, T., Y. Koyama, and T. Kondo, MK3TOOLS \& NetCDFstoring VLBI data in a machine independent array oriented data format, Proceeding 18th European VLBI for Geodesy and Astrometry (EVGA) Working Meeting April 12-13, 2007, Vienna, Austria, http://mars.hg.tuwien.ac.at/ evga/, P03, 2007.

IERS, Explanatory Supplement to IERS Bulletins A and B, http://hpiers. obspm.fr/eoppc/bul/bulb/explanatory.html, 2004.

Johnson, T., S. M. Carter, A. Myers, S. Lambert, and W. Wooden, Rapid Service/Prediction Centre, IERS Annual Report 2005, 57-66, 2005.

Kondo, T., Y. Koyama, H. Takeuchi, and M. Kimura, Current Status of the K5 Software Correlator, IVS NICT-TDC News, 25, 23-27, 2004.

Kondo, T., Y. Koyama, H. Takeuchi, and M. Kimura, Development of a New VLBI Sampler Unit (K5/VSSP32) Equipped with a USB 2.0 Interface, in IVS 2006 General Meeting Proceedings, edited by Dirk Behrend and Karen Baver, 195-199, NASA/CP-2006-214140, 2006.

Koyama, Y., T. Kondo, H. Takeuchi, M. Hirabaru, K. Takashima, D. Lapsley, K. Dudevoir, and A. R. Whitney, Rapid turn around UT1 estimation with e-VLBI, IVS NICT-TDC News, http://www2.nict.go.jp/w/ w114/stsi/ivstdc/news-index.html, 25, 28-35, 2004.

Ma, C. and D. S. Caprette, Earth orientation parameters, site positions with estimated site velocities, and source positions from the NASA Crustal Dynamics Project Using AM0-2 and two-month stepwise positions from HRAS 085, IERS Tech. Note, 11, 3-16, 1992.

Machida, M., M. Ishimoto, and K. Takashima, Tsukuba VLBI Correlator, in International VLBI Service for Geodesy and Astrometry 2005 Annual Report, edited by D. Behrend and K. Baver, 186-189, NASA/TP-2006214136, 2006.

McCarthy, D. D. and G. Petit, IERS Conventions (2003), IERS Tech. Note 32, 2004.

Meiss, M. R., Tsunami: A High-Speed Rate-Controlled Protocol for File Transfer, http://steinbeck.ucs.indiana.edu/ mmeiss/papers/tsunami.pdf, 2004.
Petrov, L., Mark-5 VLBI Analysis Software Calc/Solve, http://gemini.gsfc. nasa.gov/solve/, 2007.

Ritakari, J. and A. Mujunen, Gbit/s VLBI and eVLBI with Off-The-Shelf Components, in International VLBI Service for Geodesy and Astrometry 2004 General Meeting Proceedings, edited by Nancy R. Vandenberg and Karen D. Baver, 182-185, NASA/CP-2004-212255, 2004.

Robertson, D. S., W. E. Carter, J. Campbell, and H. Schuh, Daily earth rotation determinations from IRIS very long baseline interferometry, Nature, 316, 424-427, 1985.

Rogers, A. E. E., Very Long Baseline Interferometry with Large Effective Bandwidth for Phase-Delay Measurements, Radio Sci., 5, 1239-1247, 1970.

Tingay, S., T. Tzioumis, A. Deller, C. Phillips, S. Amy, J. Dickey, S. Ellingsen, B. Reid, S. Gulyaev, and T. Natusch, Summary of current and future e-VLBI developments in Australasia, 6th International $e$-VLBI workshop, http://www.mpifr-bonn.mpg.de/div/vlbi/6th_evlbi/, 2007.

Titov, O., V. Tesmer, and J. Boehm, OCCAM v. 6.0 software for VLBI data analysis, in International VLBI Service for Geodesy and Astrometry 2004 General Meeting Proceedings, edited by Nancy R. Vandenberg and Karen D. Baver, 267-271, NASA/CP-2004-212255, 2004.

Whitney, A. R., Future directions in VLBI technology, in EVN Symposium 2000, Proceedings of the 5th European VLBI Network Symposium held at Chalmers University of Technology, Gothenburg, Sweden, June 29July 1, 2000, edited by J. E. Conway, A. G. Polatidis, R. S. Booth, and Y. M. Pihlstrm, p. 233, 2000.

Whitney, A. R., The Mark 5B VLBI Data System, in International VLBI Service for Geodesy and Astrometry 2004 General Meeting Proceedings, edited by Nancy R. Vandenberg and Karen D. Baver, NASA/CP2004-212255, 177-181, 2004.

M. Sekido (e-mail: sekido@nict.go.jp), H. Takiguchi, Y. Koyama, T. Kondo, R. Haas, J. Wagner, J. Ritakari, S. Kurihara, and K. Kokado 